\title{
Kernos
}

Revue internationale et pluridisciplinaire de religion grecque antique

$10 \mid 1997$

Varia

\section{P. ELLINGER, La légende nationale phocidienne}

\section{Yves Lafond}

\section{(2) OpenEdition}

Journals

Édition électronique

URL : http://journals.openedition.org/kernos/688

DOI : $10.4000 /$ kernos. 688

ISSN : 2034-7871

Éditeur

Centre international d'étude de la religion grecque antique

Édition imprimée

Date de publication : 1 janvier 1997

Pagination : 354-357

ISSN : 0776-3824

Référence électronique

Yves Lafond, «P. ELLINGER, La légende nationale phocidienne », Kernos [En ligne], 10 | 1997, mis en ligne le 12 avril 2011, consulté le 24 septembre 2020. URL : http://journals.openedition.org/kernos/688 ;

DOI : https://doi.org/10.4000/kernos.688 
Pierre Elunger, La légende nationale phocidienne. Artémis, les situations extrêmes et les récits de guerre d'anéantissement, Paris, de Boccard - École française d'Athènes, 1993. 1 vol. $18,5 \times 24$ cm, 383 p., 12 pl. (BCH, Suppl. 27). ISBN : 2-86958-062-2. ISSN : 0304-2456.

En matière de religion grecque, le temps n'est plus aux volumineuses et ambitieuses synthèses du genre de celles que proposa M.P. NILSSON : les recherches menées dans les dernières décennies ont été consacrées particulièrement à mieux cerner les individualités divines et à étudier aussi bien les modes d'action des divinités que leurs divers champs d'application, tout en prenant en compte les mythes, les rites et les représentations figurées qui définissent toute expérience religieuse. Le présent volume se situe dans le sillage de ces nouvelles approches; il constitue le point d'aboutissement des travaux menés par l'A. pour un Doctorat d'État (soutenu en 1988) qui s'intitulait : Rechercbes sur les "situations extrêmes" dans la mytbologie d'Artémis et la pensée religieuse grecque. Autour de la Légende nationale pbocidienne et des récits de guerre d'anéantissement. Le nouveau titre, quoique légèrement raccourci, constitue toujours à lui seul un véritable programme de réflexion, où l'on remarquera cependant que les composantes de la problématique ont été inversées, la dimension historique et géographique semblant prendre le pas sur la dimension religieuse. Qu'on ne s'y trompe pas : histoire, géographie et histoire religieuse sont en fait étroitement associées dans un travail qui a pour sujet la guerre en Grèce ancienne aux époques archaïque et classique, mais s'attache à des cas d'exception, à des guerres hors normes qui permettent de définir une problématique des «situations extrêmes » - l'expression renvoyant aussi bien à l'idée de crise qu'à celle de confins - traitée à la lumière de deux références privilégiées, Artémis et la Phocide. Après une introduction où l'A. définit son sujet, présente sa documentation et justifie sa démarche, l'étude est organisée en trois parties d'ampleur croissante, dont les titres quelque peu énigmatiques (« Le gypse et la boue ", "Imaginaires », " Retour au monde réel ») font apparaître toutefois un cheminement qui se fonde sur une opposition entre l'imaginaire et le monde réel.

La première partie est un diptyque. Un premier chapitre, qui constitue la version revue et remaniée d'un ancien article (Le gypse et la boue. I. Sur les mytbes de la guerre d'anéantissement, in QUCC, 29 [1978], p. 7-35), s'attache à présenter les récits relatifs au conflit qui opposa les Phocidiens aux Thessaliens, dans le courant du $\mathrm{vI}^{\mathrm{e}}$ siècle av. J.-C., autour de la cité d'Hyampolis : d'Hérodote à Pausanias, il y a là un ensemble narratif dont l'élaboration peut être mise en rapport avec l'important sanctuaire d'Artémis Élaphébolos révélé par les fouilles du site de Kalapodi, un sanctuaire des confins dont l'étude contribue à éclairer l'histoire de la Phocide archaïque et permet de mieux définir l'espace où se déroulait la « Légende nationale phocidienne » (voir les utiles photographies regroupées et commentées en fin de volume). Mais, dans ces récits, ce qui retient surtout l'attention de l'A., ce sont les symétries ou oppositions qu'on peut y faire apparaître - et notamment un principe de fonctionnement essentiel, celui qui oppose la ruse à la transgression des normes, un principe qui trouve son illustration dans des épisodes où les combattants s'enduisent de boue ou de chaux pour tromper l'ennemi et rétablir un équilibre entre le combat hoplitique et la guerre d'anéantissement. En arrivant ainsi à l'opposition gypse / boue, l'A. va s'efforcer de montrer, de manière indépendante, à travers une analyse du contenu et de la valeur de ces deux termes, que cette opposition a bien existé dans la pensée grecque antique. C'est ce à quoi est consacré le deuxième chapitre, où sont mis à contribution des 
domaines très divers (monde animal, vocabulaire des techniques, récits de bataille, traditions mythiques et religieuses) et des textes très variés, allant d'Hésiode à Pausanias, en passant par Théophraste, Thucydide, Polybe ou Plutarque - sans compter les nombreuses scholies. L'A. est conscient qu'il s'agit d'un détour, mais il en souligne la valeur «stratégique » (p. 47) et d'ailleurs, ce détour prélude à un détour encore plus long, auquel l'A. va procéder dans la seconde partie.

Avant d'étudier le rôle joué par Artémis dans les récits phocidiens et d'envisager le rapport de ces récits avec la théologie d'Artémis, l'A. entend "replacer tout l'ensemble dans le cadre d'une pensée religieuse beaucoup plus vaste » (p. 91) : il s'agira d'étudier les valeurs «mythiques» du gypse et, à l'intérieur de ces valeurs, de découvrir le domaine correspondant à l'ordre de l'imaginaire. Le chapitre III nous transporte ainsi au « pays de la terre blanche ", dans la région de Sicyone et de Corinthe, un pays que sa fertilité proverbiale reliait à un passé d'âge d'or et dont les traditions mythiques (rapportées surtout par Pausanias) faisaient le lieu de naissance d'êtres ambigus, nés de la chaux, mais aussi de l'humanité véritable, née de la boue. Dans le chapitre suivant, l'analyse est centrée sur un seul texte, un extrait des Histoires d'Hérodote (III, 17-25), où il est question du pays des Éthiopiens Longue-Vie. Dans ce texte « complexe et organisé » (p. 105), l'évocation du régime alimentaire des Éthiopiens, de leur fontaine à l'odeur de violette et de leurs coutumes funéraires permet non seulement d'établir de nouveaux rapprochements avec la thématique de l'âge d'or et d'approfondir l'étude du symbolisme du gypse, mais aboutit aussi à une reflexion sur les rapports du mythe avec l'histoire. Le dernier chapitre de cette deuxième partie prend comme nouvel exemple de "situation extrême » le mythe central de la pensée orphique, construit comme on sait autour du récit du démembrement de Dionysos par les Titans. Dans cette «étrange et sinistre affaire » (p. 148), les éléments clés sont le masque de gypse que se composent les Titans pour perpétrer leur crime sans être reconnus, et la suie qui s'échappe des restes calcinés des Titans foudroyés par Zeus, suie d'où naîtra la race humaine. L'A. replace ces détails dans le contexte de la problématique anti-sacrificielle de la théologie orphique, telle qu'elle a bien été mise en valeur par M. DETIENNE (Dionysos mis à mort, Paris, 1977) et montre que c'est le système religieux traditionnel de la cité grecque, c'est-à-dire la norme elle-même, qui est dénoncé ici comme «situation extrême », selon un processus d'inversion dont l'A. s'attache à dégager le sens. En prolongement de sa démonstration, l'A. revient aux problèmes de la guerre : absence de la Titanomachie dans la théogonie orphique; liaison entre guerre et sacrifice dans le mysticisme grec (autour du traité de Porphyre, De l'abstinence); pensée et pratique pythagoriciennes de la guerre (illustrées en particulier par la guerre de Crotone contre Sybaris à l'époque archaïque).

La troisième partie est destinée à faire comprendre le rôle que joue Artémis dans les mythes de la guerre d'anéantissement. Reprenant les thèmes et les problèmes traités dans les deux parties précédentes, elle marque un retour au réel et à l'histoire, et replonge le lecteur dans les Récits phocidiens. L'objectif que se fixe d'abord l'A. (chapitre VI) est de démontrer la cohérence interne qui unit ces récits : il y parvient en reconstituant, à partir de textes d'Hérodote et de Pausanias concernant Hyampolis, mais aussi les Thermopyles, un système de quatre stratagèmes dont il montre qu'ils se répondent exactement deux à deux en une alternance d'échecs et de succès. Ayant fait apparaître la logique de cette structure narrative, l'A. s'efforce ensuite d'interpréter l'utilisation qui en est faite dans le contexte propre des récits, défini comme celui de 
l'opposition entre le combat hoplitique et la guerre d'anéantissement et où surgit « une morale de l'action et de la guerre " (p. 220). Il est alors possible d'envisager la question du rapport de ces stratagèmes et du « cycle phocidien » avec la personnalité d'Artémis et de montrer, dans un premier temps, en prenant appui sur trois exemples choisis (les chèvres d'Aigeira, la roche de Mégare et le retour de Thrasybule), que la ruse est, dans le domaine de la guerre, un mode d'action privilégié de la déesse ( $c f$. déjà P. ELLINGER, Les ruses de guerre d'Artémis, in Recbercbes sur les cultes grecs d'Occident 2, 1984 [Cabiers du Centre Jean Bérard, 9], p. 51-67). Dans un deuxième temps, qui constitue aussi le dernier chapitre du livre et le plus étendu (100 pages à lui seul), l'A. se propose

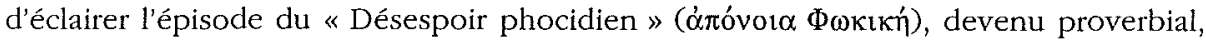
au cours duquel, avant d'engager un ultime assaut contre les Thessaliens, les Phocidiens rassemblent femmes, enfants, richesses et images des dieux sur un seul bûcher auquel il sera mis feu en cas d'échec. La version la plus développée de cette histoire est celle de Pausanias (X, 1, 4-11); mais c'est un texte de Plutarque (Vertus des femmes, $244 \mathrm{~b}-\mathrm{e}$ ) qui en dégage de la manière la plus nette la signification à la fois historique et religieuse. Récusant l'interprétation réductrice de NILSSON (qui ne voyait dans le mythe phocidien que la projection d'un rite en l'honneur d'Artémis et voulait identifier la déesse d'Hyampolis avec une Artémis Laphria), l'A. conduit donc son enquête en deux volets : l'un reprend l'étude du dossier d'Artémis Laphria, en liaison avec une analyse du mythe de Méléagre et de la chasse de Calydon (voir aussi à présent J.-M. Renaud, Le mythe de Méléagre. Essais d'interprétation, Liège, 1993); l'autre fait ressortir, à travers une confrontation entre le récit du Désespoir phocidien et des exemples historiques d'àmóvota (en particulier des suicides collectifs), la spécificité de l'épisode phocidien, en montrant qu'on peut l'interpréter comme le mythe fondateur de la Confédération phocidienne, racontant la Libération nationale de la Phocide. Dans cette réflexion, dont l'un des points d'aboutissement correspond à l'idée qu'il y eut en Phocide une « conceptualisation spécifique de la guerre » (p. 322), l'aspect religieux rejoint l'aspect politique et historique, tandis que l'histoire et la légende de la Phocide s'éclairent mutuellement, jusque dans le dernier exemple examiné par l'A., le récit des première et troisième guerres sacrées, qui permet d'aborder le cas où des peuples subissent l'anéantissement pour leur impiété, notamment dans l'espace de l'amphictionie pyléo-delphique.

$\grave{A}$ la lecture de cet ouvrage, auquel l'Association pour l'encouragement des Études grecques a décerné le prix Reinach ( $c f$. REG, 108 [1995], p. LVIII-LIX), on ne peut manquer de relever d'emblée la place privilégiée que l'A. accorde aux sources littéraires antiques: les notes infrapaginales fourmillent de références à des textes anciens de toute nature - prenant le pas d'ailleurs sur la bibliographie moderne, réduite à l'essentiel - et témoignent du souci qu'a l'A. d'appuyer ses démonstrations sur l'étude de « dossiers » exhaustifs. Cela nous vaut, dans des domaines variés, de précieuses études de vocabulaire (autour du gypse et de la chaux, à propos de l'odeur des violettes ou de la notion d'áróvot $\alpha$ par exemple), tout comme cela permet à l'A., par le rapprochement de témoignages qui avaient été négligés, traités isolément ou selon d'autres perspectives, de reprendre l'étude de quelques grandes problématiques touchant à l'histoire et à la religion de la Grèce antique : le combat hoplitique, les coutumes funéraires, les valeurs du sacrifice, l'orphisme (voir en outre Ph. BORGEAud (éd.), Orphisme et Orphée, Droz, 1991), la naissance de la cité. Certes, l'A. cède parfois à la tentation d'un style quelque peu ésotérique (une expression telle que «le bouilli des Éthiopiens est 
aphone " (p. 109) paraît, si l'on ose dire, des plus savoureuses) et, à y regarder de près, les exemples utilisés comme pièces des différents « dossiers " n'apportent peut-être pas toujours toutes les précisions souhaitables. Prenons le cas de l'épisode des « chèvres d'Aigeira », auquel nous avons eu l'occasion de nous intéresser nous-même dans le cadre de recherches sur l'Achaïe et sur Pausanias (voir Y. LAFOND, Artémis en Achaïe, in $R E G, 104$ [1991], p. 410-433) : il est regrettable que, dans la présentation de la cité d'Aigeira (p. 222), aucune mention ne soit faite des travaux de l'École autrichienne (voir W. Alzinger [et al.], Klio, 67 [1985], p. 389-451; 68 [1986], p. 5-62 et 309-347), dont les fouilles, qui se poursuivent encore actuellement sous la direction d'A. BAMMER, ont une importance équivalente à celle des recherches menées par R. FELSCH à Hyampolis et ont permis de renouveler les conclusions de FRAZER ou ANDERSON (cités p. 222, n. 109). L'Artémis de Pellène citée plus loin (p. 223) d'après Pausanias semble bien avoir été une Artémis chasseresse, mais ne porte pas l'épiclèse d'Agrotéra, comme semble le sous-entendre l'A., dans le texte du Périégète. S'agissant enfin des types monétaires d'Artémis, ne serait-il pas intéressant de signaler qu'à Aigion aussi, en Achaïe, on retrouve des représentations d'une chasseresse dadophore? Dans le cas de l'Artémis Laphria de Patras, et à propos de l'introduction de son culte par Auguste dans la nouvelle colonie, la monographie de J. HERBILlon (citée p. 246-247) n'est plus la seule référence possible : voir F. TrotTa, Il sinecismo di Patrasso in Pausania e Strabone, in $P P$ (1993), p. 428-444. Mais ces quelques réserves voudraient montrer seulement que, dans l'optique choisie par l'A., ce qui compte surtout, c'est d'utiliser la documentation au service d'une argumentation qui puisse réussir à tenir ensemble les fils de l'histoire, de l'histoire des religions et de l'imaginaire. Et, de fait, à travers les détours et retours savamment calculés dans lesquels est entraîné le lecteur, on perçoit l'enthousiasme de l'A. à construire une démonstration qui s'inscrit dans le sillage des meilleures approches anthropologiques de la Grèce ancienne (voir les travaux fréquemment cités de J.P. VERnANT et de « l'École de Paris »). Il faut donc être prudent en utilisant le double index (textes cités et index général) qui clôt ce volume : les textes et les sujets auxquels ils renvoient doivent être toujours replacés dans le mouvement de la démonstration. Dans sa préface à un ouvrage édité par Chr. Bron et E. KASSAPOGlou (L'image en jeu, de l'Antiquité à Paul Klee, Univ. de Lausanne, éd. Cabédita, 1992, p. 12), $\mathrm{Cl}$. BÉRARD faisait remarquer que, pour apprécier la richesse des systèmes iconographiques, «il existe heureusement plusieurs serrures et plusieurs clés » et ajoutait : «théoriquement, les unes ne sont pas meilleures que les autres. Elles ouvrent simplement des compartiments différents ». Une telle remarque pourrait très bien convenir, nous semble-t-il, au présent ouvrage, replacé dans le contexte des études consacrées à la personnalité d'Artémis et, plus généralement, à la guerre dans le monde grec antique.

Yves LAFOND

(Université d'Artois)

Louise-Marie L'Homme-WÉRY, La perspective éleusinienne dans la politique de Solon, Genève, Droz, 1 vol. $16 \times 24,5 \mathrm{~cm}, 391 \mathrm{p}$. (Bibliothèque de la Faculté de Pbilosophie et Lettres de l'Université de Liège, fasc. 268). ISBN Broché : 2-87019-268-1; ISBN Relié : 2-87019-068-9.

Cet ouvrage est d'un grand intérêt pour la compréhension de l'œuvre politique de Solon et pour l'exégèse aussi de son œuvre poétique. Prolongeant une thèse originale 